Pacific

Journal of

Mathematics

LEGENDRE SUMS, SOTO-ANDRADE SUMS AND KLOOSTERMAN SUMS

Anthony C. Kable 


\title{
LEGENDRE SUMS, SOTO-ANDRADE SUMS AND KLOOSTERMAN SUMS
}

\author{
Anthony C. Kable
}

\begin{abstract}
The three sums named in the title are all known to appear in connection with the complex representation theory of GL $(2, q)$. The first two are incarnations of certain spherical vectors, whereas the third is a matrix coefficient for a parabolic basis. In this work, Legendre and Soto-Andrade sums are shown to occur in a second way, as parabolic ClebschGordan coefficients for the tensor product of two Steinberg representations. This realization connects them with Kloosterman sums, and from it we derive a number of identities.
\end{abstract}

\section{Introduction.}

There is ongoing interest in special sums, the analogues over finite fields of special functions (see, for example, $[\mathbf{2}],[\mathbf{5}],[\mathbf{8}],[\mathbf{1 0}]$ ). Much of this work has focussed on identifying special sums that correspond to well-known special functions and then establishing identities for these sums that parallel classical identities. The interplay between special functions and the representation theory of classical groups, which is familiar over the real and complex numbers (see [12]), also exists over finite fields (see $[\mathbf{6}],[\mathbf{9}],[\mathbf{1 1}]$ ). It takes on a special importance in this context, since many of the other techniques of classical special functions theory (differential equations, recurrence relations and the like) do not transfer well to finite fields.

The Legendre sums have appeared in various contexts. They seem to have been christened by Evans [2], in a paper concerned with special sums analogous to classical orthogonal polynomials. In [11] they appear, in a disguised form, in a formula for the spherical vectors in a particular model of the principal series of $\operatorname{PGL}(2, q)$. This identification is attributed to GarcíaZambrano, but, whatever the provenance of these sums, Evans' name for them seems apt. The Soto-Andrade sums first appeared in [11] in a formula for the spherical vectors in a particular model of the cuspidal representations of $\operatorname{PGL}(2, q)$. They are, in a definite sense, unitary Legendre sums and were so named in an earlier version of this work. It is remarkable that, as shown in Theorem 4.1, the Legendre and Soto-Andrade sums also appear as the Clebsch-Gordan coefficients connecting the natural parabolic bases for the tensor product of two Steinberg representations. After establishing 
our notation and some elementary facts, in Section 2, and reviewing certain aspects of the representation theory of $\mathrm{GL}(2, q)$, in Section 3, we are concerned, in Sections 4,5 and 6, with exploring some of the consequences of this result.

Let $\mathbb{F}$ be a finite field of odd cardinality $q$. The Legendre and SotoAndrade sums on $\mathbb{F}$ together form a complete orthogonal basis in the inner product space $\ell^{2}(\mathbb{F}, m)$, where $m$ is the measure assigning mass $q+1$ to the points \pm 1 and mass 1 to all other points. We tentatively suggest that $\ell^{2}(\mathbb{F}, m)$ may be the appropriate finite field analogue of $L^{2}[-1,1]$. The orthogonality follows directly from the interpretation of these sums as spherical functions on $\operatorname{PGL}(2, q)$ and it is derived in this way in [3], Theorem 2.2. Our approach inevitably leads to the same result and also to a neat evaluation of the squared norms of the basis elements. This is stated as Theorem 4.2. The families of Legendre sums and Soto-Andrade sums are both indexed by characters, although of different groups, and there is a single nontrivial character that may be thought of as appearing in both families. In such a situation a Davenport-Hasse type relation is to be expected, and it is given in Theorem 4.5.

Kloosterman sums are parabolic matrix coefficients for the Steinberg representation. This implies a connection between Kloosterman sums or, more correctly, products of Kloosterman sums and Legendre and Soto-Andrade sums. This connection is explored in Sections 5 and 6. In Section 5 we study the subspace of $\ell^{2}(\mathbb{F}, m)$ spanned by the Soto-Andrade sums. It is shown that this subspace may be expressed as the nullspace of a summation operator defined using Kloosterman sums. We are led, in particular, to the following striking result. Let $p$ be a prime and

$$
S(1, a ; p)=\sum_{n=1}^{p-1} e^{2 \pi i(n+a \bar{n}) / p}
$$

be the classical Kloosterman sum, where $a \in \mathbb{Z}$ and $\bar{n}$ denotes any multiplicative inverse of $n$ modulo $p$. Abbreviate $S(1, a ; p)$ as $S(a)$. Then, for any integer $k$ not divisible by $p$, the rank of the $(p-1)$-by- $p$ matrix

$$
[S(i j) S(i(j+k))]_{i=1, \ldots,(p-1) ; j=0, \ldots,(p-1)}
$$

is exactly $(p+1) / 2$. This statement is a special case of Corollary 5.6.

We have restricted ourselves here to the case of a tensor product of Steinberg representations in order to keep the calculations within manageable bounds and to focus on a particularly interesting case. By studying more general tensor products on $\mathrm{GL}(2, q)$ it is possible to generalize a number of the results obtained here, particularly those of Sections 5 and 6 . This will be reported on elsewhere. 


\section{Notation and elementary results.}

Let $\mathbb{F}$ be a finite field with $q$ elements and $\mathbb{K}$ the unique quadratic extension of $\mathbb{F}$. We assume that $q$ is odd. Let $z \mapsto z^{\prime}$ be the nontrivial Galois automorphism of $\mathbb{K}$ over $\mathbb{F}$. Denote by $S: \mathbb{K} \rightarrow \mathbb{F}$ the trace and by $N: \mathbb{K} \rightarrow \mathbb{F}$ the norm in the extension $\mathbb{K} / \mathbb{F}$. Let $\mathcal{N}=\{z \in \mathbb{K} \mid N(z)=1\}$. If $Y$ is any set and $y \in Y$ then let $D_{y}: Y \rightarrow \mathbb{C}$ be the Kronecker delta at $y$.

For any finite abelian group $A$ let $X(A)$ denote the group of homomorphisms from $A$ to $\mathbb{C}^{\times}$. In the case where $A$ is either $\mathbb{F}^{\times}$or $\mathbb{K}^{\times}$it will be convenient to extend the elements of $X(A)$ by declaring them to be zero at zero. The trivial character in both $X\left(\mathbb{F}^{\times}\right)$and $X\left(\mathbb{K}^{\times}\right)$will be written as $\varepsilon$ and $\varphi \in X\left(\mathbb{F}^{\times}\right)$will be the quadratic character of $\mathbb{F}$. Let $X_{0}\left(\mathbb{K}^{\times}\right)=\left\{\nu \in X\left(\mathbb{K}^{\times}\right) \mid \nu(x)=1 \forall x \in \mathbb{F}^{\times}\right\}$. We call $\nu \in X\left(\mathbb{K}^{\times}\right)$indecomposable if $\nu$ is not of the form $\chi \circ N$ for any $\chi \in X\left(\mathbb{F}^{\times}\right)$. Two conditions equivalent to $\nu$ being indecomposable are that $\nu$ is not fixed under the automorphism $z \mapsto z^{\prime}$ and that the restriction of $\nu$ to $\mathcal{N}$ is nontrivial. We shall denote the set of indecomposable characters of $\mathbb{K}^{\times}$by $X^{\prime}\left(\mathbb{K}^{\times}\right)$. In contrast, $X^{\prime}\left(\mathbb{F}^{\times}\right)$will denote the set $X\left(\mathbb{F}^{\times}\right) \backslash\{\varepsilon, \varphi\}$. Finally, $X_{0}^{\prime}\left(\mathbb{K}^{\times}\right)$will be the set of indecomposable characters of $\mathbb{K}^{\times}$that are trivial on $\mathbb{F}^{\times}$.

Fix, once and for all, a nontrivial character $\psi \in X(\mathbb{F})$. For $\chi \in X\left(\mathbb{F}^{\times}\right)$let

$$
\Gamma(\chi)=\sum_{x \in \mathbb{F}} \chi(x) \psi(x)
$$

be the corresponding Gauss sum. For later use we recall the reflection formula

$$
\Gamma(\chi) \Gamma\left(\chi^{-1}\right)=q \chi(-1)-(q-1) D_{\varepsilon}(\chi) .
$$

We will refer to Greene's analogues of hypergeometric functions over finite fields, as described in [5]. For the reader's convenience we give the definition in the simplest case. For characters $\chi_{0}, \chi_{1}, \chi_{2} \in X\left(\mathbb{F}^{\times}\right)$and $x \in \mathbb{F}$, Greene defines

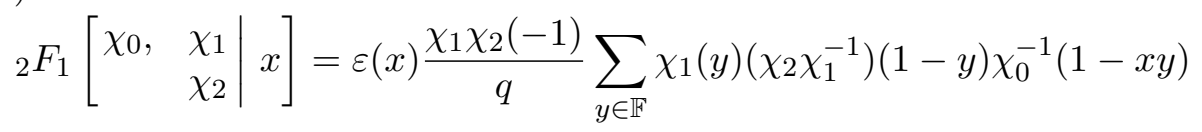

and proves that this sum satisfies analogues of many familiar properties of the classical ${ }_{2} F_{1}$.

We now recall the definitions of the various character sums. These sums may be regarded as complex-valued functions on $\mathbb{F}$ and, throughout, we let $a \in \mathbb{F}$. For $\chi \in X\left(\mathbb{F}^{\times}\right)$, the generalized Kloosterman sum of order $\chi$ is

$$
K_{\chi}(a)=\frac{1}{q} \sum_{x \in \mathbb{F}^{\times}} \chi(x) \psi\left(x+\frac{a}{x}\right) .
$$


We shall write $K$ instead of $K_{\varepsilon}$ in the case that the order is the trivial character. Note that our definition includes a factor of $1 / q$ that does not appear in the standard definition; it is inserted to simplify subsequent formulas. For this reason, and to emphasize the analogy with classical Bessel functions, we avoid the standard notation for Kloosterman sums. For $\chi \in X\left(\mathbb{F}^{\times}\right)$, the Legendre sum of order $\chi$ is

$$
P_{\chi}(a)=\frac{1}{q} \sum_{x \in \mathbb{F}^{\times}} \chi(x) \varphi\left(1-2 a x+x^{2}\right) .
$$

Finally, for $\nu \in X_{0}\left(\mathbb{K}^{\times}\right)$, the Soto-Andrade sum of order $\nu$ is

$$
R_{\nu}(a)=\frac{1}{q(q-1)} \sum_{z \in \mathbb{K}^{\times}} \nu(z) \varphi\left(S(z)^{2}-2(a+1) N(z)\right) .
$$

It is easy to see that the Soto-Andrade sums may be reexpressed as

$$
R_{\nu}(a)=\frac{1}{q} \sum_{\zeta \in \mathcal{N}} \omega_{\nu \cdot(\varphi \circ N)}(\zeta) \varphi\left(\zeta-2 a+\zeta^{-1}\right) .
$$

A comparison of this and the expression

$$
P_{\chi}(a)=\frac{1}{q} \sum_{x \in \mathbb{F}^{\times}}(\chi \varphi)(x) \varphi\left(x-2 a+x^{-1}\right)
$$

reveals the close analogy between the two sums.

The following lemmas list some elementary properties of the Legendre and Soto-Andrade sums. They can be proved with ease directly from the definitions and the alternate expressions (3) and (4).

Lemma 2.1. The values of the Legendre sum and Soto-Andrade sum of order $\varepsilon$ are

$$
P_{\varepsilon}(a)=\left\{\begin{array}{cl}
\frac{q-2}{q} & \text { if } a= \pm 1 \\
-\frac{2}{q} & \text { if } a \neq \pm 1
\end{array}\right.
$$

and

$$
R_{\varepsilon}(a)= \begin{cases}-1 & \text { if } a=1, \\ 1 & \text { if } a=-1, \\ 0 & \text { if } a \neq \pm 1\end{cases}
$$

Lemma 2.2. Suppose that $\chi \in X\left(\mathbb{F}^{\times}\right), \nu \in X_{0}\left(\mathbb{K}^{\times}\right)$and neither $\chi$ nor $\nu$ is trivial. Then $P_{\chi}(1)=-1 / q$ and $R_{\nu}(1)=1 / q$.

Lemma 2.3. For $\chi \in X\left(\mathbb{F}^{\times}\right), \nu \in X_{0}\left(\mathbb{K}^{\times}\right)$and $a \in \mathbb{F}$ we have $P_{\chi^{-1}}(a)$ $=P_{\chi}(a), \quad P_{\chi}(-a)=\chi(-1) P_{\chi}(a), \quad R_{\nu^{-1}}(a)=R_{\nu}(a) \quad$ and $\quad R_{\nu}(-a)=$ $-\nu\left(n^{1 / 2}\right) R_{\nu}(a)$, where $n \in \mathbb{F}$ is any element that satisfies $\varphi(n)=-1$. 
From Lemma 2.2 and Lemma 2.3 we obtain the evaluations

$$
P_{\chi}(-1)=-\frac{\chi(-1)}{q}, \quad R_{\nu}(-1)=-\frac{\nu\left(n^{1 / 2}\right)}{q},
$$

where $n \in \mathbb{F}$ satisfies $\varphi(n)=-1$ and $\chi$ and $\nu$ are nontrivial. For use in the proof of Theorem 4.1, we need to observe a second expression for $R_{\nu}(-1)$ when $\nu \neq \varepsilon$. The definition of $R_{\nu}(-1)$ gives

$$
R_{\nu}(-1)=\frac{1}{q(q-1)} \sum_{\substack{z \in \mathbb{K}^{\times} \\ S(z) \neq 0}} \nu(z)=-\frac{1}{q(q-1)} \sum_{\substack{z \in \mathbb{K}^{\times} \\ S(z)=0}} \nu(z) .
$$

In $[\mathbf{1 0}]$ it is shown that the Legendre sum $P_{\chi}$ may be expressed in terms of Greene's hypergeometric sum. However, the expression offered in [10] does not correspond to the most usual expression in the classical case and has an awkward extra term. In Proposition 2.5 we give a different expression, closely parallel to the standard one and with no additional terms. Lemma 2.4 will be used repeatedly in what follows.

Lemma 2.4. Suppose that $A \in \mathbb{F}^{\times}$and $B \in \mathbb{F}$. Then

$$
\sum_{x \in \mathbb{F}} K\left(A x^{2}\right) \psi(B x)=\varphi\left(B^{2}-4 A\right) .
$$

Proof. We begin by considering $S=\sum_{x \in \mathbb{F}^{\times}} K\left(A x^{2}\right) \psi(B x)$. Introducing the definition of $K\left(A x^{2}\right)$ as a sum over the variable $y$, replacing $y$ by $x y$ and then replacing $x$ by $y x$ we obtain $S=\frac{1}{q} \sum_{x, y \in \mathbb{F}} \times\left(\left(y^{2}+B y+A\right) x\right)$. Evaluating the sum over $x$ gives

$$
\begin{aligned}
S & =\frac{1}{q} \sum_{\substack{y \in \mathbb{F}^{\times} \\
y^{2}+B y+A \neq 0}}(-1)+\frac{1}{q} \sum_{\substack{y \in \mathbb{F}^{\times} \\
y^{2}+B y+A=0}}(q-1) \\
& =-\frac{1}{q}(q-1)+{ }^{\#}\left\{y \in \mathbb{F}^{\times} \mid y^{2}+B y+A=0\right\} \\
& =\frac{1}{q}+\varphi\left(B^{2}-4 A\right) .
\end{aligned}
$$

The lemma follows on noting that $K(0)=-1 / q$.

Proposition 2.5. If $\chi \in X\left(\mathbb{F}^{\times}\right)$is nontrivial and $a \in \mathbb{F} \backslash\{ \pm 1\}$ then

$$
P_{\chi}(a)={ }_{2} F_{1}\left[\begin{array}{cc|c}
\chi, & \chi^{-1} & \frac{1}{2}(1-a)
\end{array}\right] .
$$

Proof. Consider the sum

$$
S=\frac{1}{q} \sum_{x, c \in \mathbb{F}^{\times}} \chi(c) K\left(\frac{1}{2}(1+a) c x^{2}\right) \psi((1+c) x) .
$$


Using Lemma 2.4 to evaluate the sum over $x$ we easily obtain $S=P_{\chi}(a)$. Before evaluating the sum in another way, we make a simple observation. If we have a function $f: \mathbb{F}^{\times} \rightarrow \mathbb{F}$ and $\mu \in X\left(\mathbb{F}^{\times}\right)$is nontrivial then $\sum_{x, c \in \mathbb{F}^{\times}} \mu(c) \psi(f(x) c)=\Gamma(\mu) \sum_{x \in \mathbb{F}^{\times}} \mu^{-1}(f(x))$, regardless of the fact that $f$ may sometimes vanish. This is easily verified by separating the terms with $f(x)=0$ from those with $f(x) \neq 0$ and recalling the convention that $\mu^{-1}(0)=0$.

Introducing the definition of $K$ with $y$ as the summation variable and then replacing $y$ by $x y$ we obtain

$$
S=\frac{1}{q^{2}} \sum_{x, y, c \in \mathbb{F}^{\times}} \chi(c) \psi\left(\frac{1+a+2 y}{2 y} c x\right) \psi((1+y) x)
$$

and using the observation of the previous paragraph twice to sum first over $c$ and then over $x$ gives

$$
S=\frac{\Gamma(\chi) \Gamma\left(\chi^{-1}\right)}{q^{2}} \sum_{y \in \mathbb{F}^{\times}} \chi(2 y) \chi(1+y) \chi^{-1}(1+a+2 y) .
$$

Since $\chi$ is nontrivial, we have $\Gamma(\chi) \Gamma\left(\chi^{-1}\right)=q \chi(-1)$ by (1). Extending the sum over all of $\mathbb{F}$, substituting $y-1$ for $y$, excluding 0 from the resulting summation, substituting $1 / y$ for $y$ and finally completing the summation again we obtain

$$
S=\frac{\chi(-1)}{q} \sum_{y \in \mathbb{F}} \chi^{-1}(y) \chi(1-y) \chi^{-1}\left(1-\frac{1}{2}(1-a) y\right) .
$$

Comparing this with (2) gives the result.

\section{Facts from representation theory.}

In this section we recall certain facts from the representation theory of the group $G=\mathrm{GL}(2, \mathbb{F})$. The majority of these facts are established in [9], to which the reader is referred for a fuller discussion. The notation introduced here will be used without comment in subsequent sections.

We begin by describing $\mathcal{C}$, the set of conjugacy classes in $G$, and a parametrization of each $c \in \mathcal{C}$. For $\alpha, \delta \in \mathbb{F}^{\times}, \beta \in \mathbb{F}$ and $z \in \mathbb{K}$ we let

$$
\begin{array}{ll}
\mathbf{w}=\left(\begin{array}{rr}
0 & 1 \\
-1 & 0
\end{array}\right), & \mathbf{t}(\alpha, \delta)=\left(\begin{array}{cc}
\alpha & 0 \\
0 & \delta
\end{array}\right), \\
\mathbf{n}(\beta)=\left(\begin{array}{ll}
1 & \beta \\
0 & 1
\end{array}\right), & \mathbf{k}(z)=\left(\begin{array}{rr}
0 & -N(z) \\
1 & S(z)
\end{array}\right) .
\end{array}
$$

For any $g \in G$ let $C(g)$ denote the conjugacy class of $g$. The conjugacy classes in $G$ come in four kinds. First there are the central conjugacy classes $C(\mathbf{t}(\alpha, \alpha))$ for $\alpha \in \mathbb{F}^{\times}$, each of which is a singleton. Secondly there are the 
unipotent conjugacy classes $C\left(\mathbf{t}(\alpha, \alpha) \mathbf{n}\left(\alpha^{-1}\right)\right)$, each of which has $(q+1)(q-$ 1) elements and may be parametrized as

$$
\left\{\left(\begin{array}{cc}
\alpha-x & -b^{-1} x^{2} \\
b & \alpha+x
\end{array}\right) \mid b \in \mathbb{F}^{\times}, x \in \mathbb{F}\right\} .
$$

Thirdly, there are the split semisimple conjugacy classes $C(\mathbf{t}(\alpha, \delta))$ for $\alpha, \delta \in$ $\mathbb{F}^{\times}$with $\alpha \neq \delta$. Since $\mathbf{t}(\alpha, \delta)$ lies in the same class as $\mathbf{t}(\delta, \alpha)$ it is convenient to introduce the equivalence relation $(\alpha, \delta) \sim(\delta, \alpha)$ on $\mathbb{F}^{\times} \times \mathbb{F}^{\times}$. If we set

$$
\Omega^{+}=\left\{(\alpha, \delta) \in \mathbb{F}^{\times} \times \mathbb{F}^{\times} \mid \alpha \neq \delta\right\}
$$

then this family of conjugacy classes is parametrized by the set $\Omega^{+} / \sim$. Each of these conjugacy classes has $q(q+1)$ elements and may be parametrized as

$$
\left\{\left(\begin{array}{ll}
\alpha & x \\
0 & \delta
\end{array}\right) \mid x \in \mathbb{F}\right\} \bigcup\left\{\left(\begin{array}{cc}
\delta+x y & (\alpha-\delta-x y) y \\
x & \alpha-x y
\end{array}\right) \mid x, y \in \mathbb{F}\right\} .
$$

Lastly, there are the non-split semisimple conjugacy classes $C(\mathbf{k}(z))$ for $z \in \mathbb{K} \backslash \mathbb{F}$. Since $\mathbf{k}(z)$ lies in the same conjugacy class as $\mathbf{k}\left(z^{\prime}\right)$ it is convenient to introduce the equivalence relation $z \sim z^{\prime}$ on the set $\Omega^{-}=\mathbb{K} \backslash \mathbb{F}$. Then this family of conjugacy classes is parametrized by the set $\Omega^{-} / \sim$. Each of these conjugacy classes has $q(q-1)$ elements and may be parametrized as

$$
\left\{\left(\begin{array}{cc}
x & -a^{-1}\left(x^{2}+N(z)-x S(z)\right) \\
a & -x+S(z)
\end{array}\right) \mid a \in \mathbb{F}^{\times}, x \in \mathbb{F}\right\} .
$$

Next we recall the list of irreducible complex representations of $G$ and introduce our notation for them. They also come in four families. First, there are the one-dimensional representations $\chi \circ \operatorname{det}$ for $\chi \in X\left(\mathbb{F}^{\times}\right)$. Secondly, there are the Steinberg representations $\pi(\chi)$, one for each $\chi \in X\left(\mathbb{F}^{\times}\right)$. Each of these has dimension $q$ and $\pi\left(\chi_{1}\right) \cong \pi\left(\chi_{2}\right)$ if and only if $\chi_{1}=\chi_{2}$. Thirdly, there are the principal series representations $\pi\left(\chi_{1}, \chi_{2}\right)$ for $\chi_{1}, \chi_{2} \in X\left(\mathbb{F}^{\times}\right)$. These all have dimension $q+1$ and are irreducible exactly when $\chi_{1} \neq \chi_{2}$. Furthermore, $\pi\left(\chi_{1}, \chi_{2}\right) \cong \pi\left(\chi_{2}, \chi_{1}\right)$ and this gives the only equivalences within this family. It is also convenient to note that $\pi(\chi, \chi) \cong \pi(\chi) \oplus(\chi \circ$ det $)$ for all $\chi \in X\left(\mathbb{F}^{\times}\right)$. Finally, there are the cuspidal representations $\rho(\nu)$ for $\nu$ an indecomposable element of $X\left(\mathbb{K}^{\times}\right)$. These are all irreducible and have dimension $q-1$. We have $\rho(\nu) \cong \rho\left(\nu^{\prime}\right)$, where $\nu^{\prime}(z)=\nu\left(z^{\prime}\right)$, and this gives the only equivalences within this family.

We shall require a fragment of the character table of $G$, displaying the character values for all irreducible representations having trivial central character, and this is given in Table 1. In the table $\mu \in\{\varepsilon, \varphi\}, \chi \in X\left(\mathbb{F}^{\times}\right)$and $\nu \in X_{0}\left(\mathbb{K}^{\times}\right)$. Note that the character values of $\pi\left(\chi, \chi^{-1}\right)$ are correct as given even when $\chi$ is $\varepsilon$ or $\varphi$ so that the corresponding principal series representation is reducible. 


\begin{tabular}{|c|c|c|c|c|}
\multicolumn{2}{r}{} & \multicolumn{1}{c}{$(q-1)(q+1)$} & $q(q+1)$ & $q(q-1)$ \\
$\mathbf{t}(\alpha, \alpha)$ & $\mathbf{t}(\alpha, \alpha) \mathbf{n}\left(\alpha^{-1}\right)$ & $\mathbf{t}(\alpha, \delta)$ & $\mathbf{k}(z)$ \\
\hline$\pi(\mu)$ & $q$ & 0 & $\mu(\alpha \delta)$ & $-\mu \circ N(z)$ \\
\hline$\pi\left(\chi, \chi^{-1}\right)$ & $(q+1)$ & 1 & $\chi\left(\frac{\alpha}{\delta}\right)+\chi\left(\frac{\delta}{\alpha}\right)$ & 0 \\
\hline$\rho(\nu)$ & $(q-1)$ & -1 & 0 & $-\nu(z)-\nu\left(z^{\prime}\right)$ \\
\hline
\end{tabular}

Table 1. A fragment of the character table of $G$.

In [9] each cuspidal representation of $G$ is constructed by giving an action of $G$ on the space of functions on $\mathbb{F}^{\times}$that affords the required representation. The matrix coefficients in this model of $\rho(\nu)$ involve unitary Kloosterman sums. Although it is not done in [9], it is possible to give similar models of the other higher-dimensional (i.e., not one-dimensional) representations of $G$ and the matrix coefficients in these models involve generalized Kloosterman sums. We shall require such a model of the Steinberg representation $\pi(\varepsilon)$. Since the correponding models over local fields are available in the literature (see [4]), we shall not offer any further explanation.

Let $W$ be a complex $q$-dimensional vector space with a basis consisting of the set of symbols $\left\{\xi^{a} \mid a \in \mathbb{F}\right\}$. Let $G$ act on $W$ according to the following formulas, where $a \in \mathbb{F}, c \in \mathbb{F}^{\times}$and $\gamma \in \mathbb{F}^{\times}$:

$$
\begin{aligned}
& \left(\begin{array}{cc}
\alpha & \beta \\
0 & \delta
\end{array}\right) \xi^{a}=\psi\left(\alpha^{-1} \beta a\right) \xi^{\alpha^{-1} \delta a} \\
& \left(\begin{array}{ll}
\alpha & \beta \\
\gamma & \delta
\end{array}\right) \xi^{0}=-\frac{1}{q} \xi^{0}-\frac{q+1}{q} \sum_{b \in \mathbb{F}^{\times}} \psi\left(\frac{\alpha b}{\gamma}\right) \xi^{b} \\
& \left(\begin{array}{ll}
\alpha & \beta \\
\gamma & \delta
\end{array}\right) \xi^{c}=-\frac{1}{q} \psi\left(\frac{\delta c}{\gamma}\right) \xi^{0}+\sum_{b \in \mathbb{F}^{\times}} K\left(\frac{b c \operatorname{det}(g)}{\gamma^{2}}\right) \psi\left(\frac{\alpha b+\delta c}{\gamma}\right) \xi^{b} .
\end{aligned}
$$

Then $W$, with this action, is a model of the representation $\pi(\varepsilon)$ and the Hermitian form defined on $W$ by

$$
\left\langle\xi^{a}, \xi^{b}\right\rangle=D_{a}(b)+q D_{0}(a) D_{0}(b)
$$

is invariant under $G$.

Put $\Pi=\pi(\varepsilon) \otimes \pi(\varepsilon)$. Using Table 1 it is easy to verify the following decomposition of $\Pi$ into irreducible constituents:

$$
\Pi \cong(\varepsilon \circ \text { det }) \oplus \pi(\varepsilon) \oplus \pi(\varphi) \oplus \bigoplus_{\chi \in X^{\prime}\left(\mathbb{F}^{\times}\right) / \sim} \pi\left(\chi, \chi^{-1}\right) \oplus \bigoplus_{\nu \in X_{0}^{\prime}\left(\mathbb{K}^{\times}\right) / \sim} \rho(\nu)
$$


The equivalence relation on $X^{\prime}\left(\mathbb{F}^{\times}\right)$is $\chi \sim \chi^{-1}$ and the equivalence relation on $X_{0}^{\prime}\left(\mathbb{K}^{\times}\right)$is $\nu \sim \nu^{\prime}$. Observe that this decomposition is multiplicity-free. For any irreducible representation $\pi$ of $G$ let $V_{\pi}$ be the subspace of $W \otimes W$ on which it is realized (with $V_{\pi}=\{0\}$ if $\pi$ does not occur).

For any representation $\pi$ of $G$ let $\operatorname{ch}_{\pi}$ be the character of $\pi$ and for any $c \in \mathcal{C}$ let $\Pi(c)=\sum_{g \in c} \Pi(g)$, where the sum is taken inside the endomorphism algebra of $W \otimes W$. If $\pi$ is irreducible then the projection operator from $W \otimes W$ onto the subspace $V_{\pi}$ is given by

$$
\mathbf{P}_{\pi}=\frac{\operatorname{deg}(\pi)}{|G|} \sum_{c \in \mathcal{C}} \overline{\operatorname{ch}_{\pi}(c)} \Pi(c),
$$

where $\operatorname{deg}(\pi)$ is the dimension of the space of $\pi$, and we may use (11) to define an operator $\mathbf{P}_{\pi}$ even when $\pi$ is reducible. It will be convenient to observe the formulas

$$
\mathbf{P}_{\pi(\varepsilon)}=-q \mathbf{P}_{\varepsilon \circ \operatorname{det}}+\frac{q}{q+1} \mathbf{P}_{\pi(\varepsilon, \varepsilon)}
$$

and

$$
\mathbf{P}_{\pi(\varphi)}=\frac{q}{q+1} \mathbf{P}_{\pi(\varphi, \varphi)},
$$

the latter because $\mathbf{P}_{\varphi \circ d e t}=0$.

The subgroup $N=\{\mathbf{n}(\beta) \mid \beta \in \mathbb{F}\}$ of $G$ is abelian and hence the space of any representation of $G$ decomposes into the sum of simultaneous eigenspaces for $N$. For $a \in \mathbb{F}$ let $\psi_{a} \in X(N)$ be the character $\psi_{a}(\mathbf{n}(\beta))=$ $\psi(a \beta)$. It is shown in [9] that if $\pi$ is a higher-dimensional irreducible representation of $G$ then the $\psi_{a}$-eigenspace of $N$ in the space of $\pi$ is exactly one-dimensional for each $a \in \mathbb{F}^{\times}$. The $\psi_{0}$-eigenspace may either be zero (if $\pi$ is cuspidal), one-dimensional (if $\pi$ is Steinberg) or two-dimensional (otherwise). We note that the vector $\xi^{a} \in W$ is a $\psi_{a}$-eigenvector for $N$. In general, we shall refer to a basis for the space of a representation of $G$ consisting of simultaneous eigenvectors for $N$ as a parabolic basis.

\section{Legendre sums as Clebsch-Gordan coefficients.}

In $W \otimes W$ we have two natural bases, one consisting of simple tensors of the form $\xi^{a} \otimes \xi^{b}$ and the other being the union of parabolic bases for each of the isotypic subspaces. We shall show that the Legendre and Soto-Andrade sums occur as entries in the change-of-basis matrix between these two bases. Such matrix entries are usually referred to as Clebsch-Gordan coefficients. In light of the results sketched in [12], Section 8.7, the appearance of Legendre functions here should not be unexpected. From this we shall deduce the main result of this section, Theorem 4.2, as well as a Davenport-Hasse type relation between the sums $R_{\varphi \circ N}$ and $P_{\varphi}$, Theorem 4.5. 
Theorem 4.1. For $v \neq 0, \chi \in X^{\prime}\left(\mathbb{F}^{\times}\right)$and $\nu \in X_{0}^{\prime}\left(\mathbb{K}^{\times}\right)$we have

$$
\begin{aligned}
& \mathbf{P}_{\varepsilon \text { odet }}\left(\xi^{0} \otimes \xi^{v}\right)=0 \\
& \mathbf{P}_{\pi(\varepsilon)}\left(\xi^{0} \otimes \xi^{v}\right)=-\frac{1}{q-1} \sum_{s \in \mathbb{F}}\left(P_{\varepsilon}(s)-\frac{q-1}{q}\right) \xi^{v\left(\frac{1-s}{2}\right)} \otimes \xi^{v\left(\frac{1+s}{2}\right)} \\
& \mathbf{P}_{\pi(\varphi)}\left(\xi^{0} \otimes \xi^{v}\right)=-\frac{q}{q-1} \sum_{s \in \mathbb{F}} P_{\varphi}(s) \xi^{v\left(\frac{1-s}{2}\right)} \otimes \xi^{v\left(\frac{1+s}{2}\right)} \\
& \mathbf{P}_{\pi\left(\chi, \chi^{-1}\right)}\left(\xi^{0} \otimes \xi^{v}\right)=-\frac{q+1}{q-1} \sum_{s \in \mathbb{F}} P_{\chi}(s) \xi^{v\left(\frac{1-s}{2}\right)} \otimes \xi^{v\left(\frac{1+s}{2}\right)} \\
& \mathbf{P}_{\rho(\nu)}\left(\xi^{0} \otimes \xi^{v}\right)=\sum_{s \in \mathbb{F}} R_{\nu}(s) \xi^{v\left(\frac{1-s}{2}\right)} \otimes \xi^{v\left(\frac{1+s}{2}\right)} .
\end{aligned}
$$

Proof. The proof is a computation. We shall give only enough details that the interested reader can reproduce it. The necessary ingredients are the formula (11) for the projection operators on $W \otimes W$, the characters of the various representations as given in Table 1, the formulas (9) for the action of $G$ on $W$, the parametrizations of the conjugacy classes in $G$ given at the beginning of Section 3 and Lemma 2.4.

The first step is to calculate $\Pi(c)\left(\xi^{0} \otimes \xi^{v}\right)$ for each $c \in \mathcal{C}$. For $c=$ $C(\mathbf{t}(\alpha, \alpha))$ we trivially obtain

$$
\Pi(c)\left(\xi^{0} \otimes \xi^{v}\right)=\xi^{0} \otimes \xi^{v} .
$$

Next we consider $c=C\left(\mathbf{t}(\alpha, \alpha) \mathbf{n}\left(\alpha^{-1}\right)\right)$. Using (9) to calculate the action of each element of the set (6) on $\xi^{0} \otimes \xi^{v}$ and then summing over $x$ and $b$ we obtain

$$
\begin{aligned}
& \Pi(c)\left(\xi^{0} \otimes \xi^{v}\right)=-\xi^{0} \otimes \xi^{v}-\sum_{b \in \mathbb{F}^{\times}} K\left(\alpha^{2} b^{2}\right) \psi(2 \alpha b) \xi^{0} \otimes \xi^{v} \\
& +\frac{q+1}{q} \sum_{b \in \mathbb{F}^{\times}} \psi(2 \alpha b) \xi^{v} \otimes \xi^{0} \\
& -(q+1) \sum_{s \in \mathbb{F} \backslash\{0,1\}} \sum_{b \in \mathbb{F}^{\times}} K\left(s \alpha^{2} b^{2}\right) \psi(2 \alpha b) \xi^{v(1-s)} \otimes \xi^{v s} .
\end{aligned}
$$

Using Lemma 2.4 to calculate the sums involving Kloosterman sums, this simplifies to

$$
\Pi(c)\left(\xi^{0} \otimes \xi^{v}\right)=\Xi-(q+1) \sum_{s \in \mathbb{F} \backslash\{0,1\}} \varphi(4(1-s)) \xi^{v(1-s)} \otimes \xi^{v s},
$$

where we have introduced the convenient abbreviation

$$
\Xi=-\frac{q+1}{q} \sum_{s \in \mathbb{F}} \xi^{v(1-s)} \otimes \xi^{v s} .
$$


The calculation for $c=C(\mathbf{t}(\alpha, \delta))$ is similar, using (7), and we obtain

$$
\begin{aligned}
\Pi(c)\left(\xi^{0} \otimes \xi^{v}\right)= & \Xi+(q+1) D_{0}(\alpha+\delta) \xi^{v} \otimes \xi^{0} \\
& -(q+1) \sum_{s \in \mathbb{F} \backslash\{0,1\}} \varphi\left(\alpha^{2}+\delta^{2}-2(2 s-1) \alpha \delta\right) \xi^{v(1-s)} \otimes \xi^{v s} .
\end{aligned}
$$

Finally, for the conjugacy class $c=C(\mathbf{k}(z))$ we get

$$
\begin{aligned}
\Pi(c)\left(\xi^{0} \otimes \xi^{v}\right)=\Xi+ & 2 \xi^{0} \otimes \xi^{v}+(q+1) D_{0}(S(z)) \xi^{v} \otimes \xi^{0} \\
& -(q+1) \sum_{s \in \mathbb{F} \backslash\{0,1\}} \varphi\left(S(z)^{2}-4 s N(z)\right) \xi^{v(1-s)} \otimes \xi^{v s} .
\end{aligned}
$$

Using (11) it is now possible to find the various projections of $\xi^{0} \otimes \xi^{v}$. In light of (12), (13) and the fact that $\mathbf{P}_{\varepsilon \text { odet }}\left(\xi^{0} \otimes \xi^{v}\right)=0$, it is sufficient to determine $\mathbf{P}_{\pi\left(\chi, \chi^{-1}\right)}\left(\xi^{0} \otimes \xi^{v}\right)$ for $\chi \in X\left(\mathbb{F}^{\times}\right)$and $\mathbf{P}_{\rho(\nu)}\left(\xi^{0} \otimes \xi^{v}\right)$ for $\nu \in$ $X_{0}^{\prime}\left(\mathbb{K}^{\times}\right)$. As an example of the shape of the calculations, we determine the coefficient of the vector $\xi^{v} \otimes \xi^{0}$ in the projection $\mathbf{P}_{\rho(\nu)}\left(\xi^{0} \otimes \xi^{v}\right)$. Following the description given above, we at first obtain

$$
\begin{aligned}
\frac{1}{q(q-1)(q+1)}\left[(q-1) \frac{q+1}{q}\right. & +\frac{q+1}{q} \sum_{z \in \Omega^{-} / \sim}\left(\nu(z)+\nu\left(z^{\prime}\right)\right) \\
& \left.-(q+1) \sum_{z \in \Omega^{-} / \sim}\left(\nu(z)+\nu\left(z^{\prime}\right)\right) D_{0}(S(z))\right]
\end{aligned}
$$

for this coefficient. Unfolding the equivalence relations in the sums, this is equal to

$$
\frac{1}{q(q-1)}\left[\frac{q-1}{q}+\frac{1}{q} \sum_{z \in \mathbb{K} \backslash \mathbb{F}} \nu(z)-\sum_{z \in \mathbb{K} \backslash \mathbb{F}} \nu(z) D_{0}(S(z))\right] .
$$

Completing the sums and using the fact that $\nu$ is nontrivial on $\mathbb{K}^{\times}$but trivial on $\mathbb{F}^{\times}$, we see that the first two terms cancel and what remains is

$$
-\frac{1}{q(q-1)} \sum_{\substack{z \in \mathbb{K}^{\times} \\ S(z)=0}} \nu(z)
$$

According to (5), this is equal to $R_{\nu}(-1)$, as claimed.

Let $m: \mathbb{F} \rightarrow \mathbb{C}$ be $m(x)=1+q D_{1}(x)+q D_{-1}(x)$ and $\ell^{2}(\mathbb{F}, m)$ be the space of complex-valued functions on $\mathbb{F}$ equipped with the Hermitian form

$$
\left\langle F_{1}, F_{2}\right\rangle=\sum_{x \in \mathbb{F}} F_{1}(x) \overline{F_{2}(x)} m(x) .
$$


As remarked in the introduction, the orthogonality of the set $\mathcal{L}$, defined below, in $\ell^{2}(\mathbb{F}, m)$ has been observed by Evans in $[\mathbf{3}]$.

Theorem 4.2. The set

$$
\mathcal{L}=\left\{P_{\varepsilon}-\frac{q-1}{q}, P_{\varphi}, P_{\chi}, R_{\nu} \mid \chi \in X^{\prime}\left(\mathbb{F}^{\times}\right) / \sim, \nu \in X_{0}^{\prime}\left(\mathbb{K}^{\times}\right) / \sim\right\}
$$

is an orthogonal basis for the space $\ell^{2}(\mathbb{F}, m)$. The square norms of the elements of this basis are as follows:

$$
\begin{aligned}
& \left\|P_{\varepsilon}-\frac{q-1}{q}\right\|^{2}=\frac{q^{2}-1}{q} \\
& \left\|P_{\varphi}\right\|^{2}=\frac{q^{2}-1}{q^{2}} \\
& \left\|P_{\chi}\right\|^{2}=\frac{q-1}{q} \\
& \left\|R_{\nu}\right\|^{2}=\frac{q+1}{q} .
\end{aligned}
$$

Proof. Equip $W \otimes W$ with the Hermitian form induced on it by the Hermitian form on $W$ satisfying (10). Note that this is a $G$-invariant form on $W \otimes W$. If $\pi_{1}$ and $\pi_{2}$ are distinct irreducible constituents of $\Pi$ then $V_{\pi_{1}} \perp V_{\pi_{2}}$ and thus the inner product of any two distinct vectors from the list (14) is zero. Now

$$
\left\langle\xi^{\frac{1-s}{2}} \otimes \xi^{\frac{1+s}{2}}, \xi^{\frac{1-t}{2}} \otimes \xi^{\frac{1+t}{2}}\right\rangle=D_{t}(s) m(s)
$$

and so, evaluating the inner products of distinct vectors on the list (14) directly for $v=1$, we obtain the orthogonality of distinct elements of $\mathcal{L}$ in $\ell^{2}(\mathbb{F}, m)$.

Every vector in $W \otimes W$ is the sum of its projections onto all the isotypic subspaces. Thus $\xi^{0} \otimes \xi^{1}$ is the sum of the vectors on the list (14) for $v=1$. Comparing coefficients on both sides of this relationship we obtain

$$
\begin{aligned}
D_{1}(s)=-\frac{1}{q-1}\left(P_{\varepsilon}(s)-\frac{q-1}{q}\right)-\frac{q}{q-1} P_{\varphi}(s) & \\
& -\frac{q+1}{q-1} \sum_{\chi \in X^{\prime}\left(\mathbb{F}^{\times}\right) / \sim} P_{\chi}(s)+\sum_{\nu \in X_{0}^{\prime}\left(\mathbb{K}^{\times}\right) / \sim} R_{\nu}(s)
\end{aligned}
$$

for all $s \in \mathbb{F}$. Multiplying this identity on both sides by $f(s) m(s)$ for any $f \in \mathcal{L}$, summing over $s \in \mathbb{F}$ and using the orthogonality of $\mathcal{L}$ on the right and Lemmas 2.1 and 2.2 on the left, we obtain the value of $\|f\|^{2}$.

Finally, to see that the orthogonal set $\mathcal{L}$ is a basis for $\ell^{2}(\mathbb{F}, m)$ we simply count; there are $2+\frac{1}{2}(q-3)+\frac{1}{2}(q-1)=q$ elements in $\mathcal{L}$ and this is the dimension of $\ell^{2}(\mathbb{F}, m)$. 
Corollary 4.3. For all $s, t \in \mathbb{F}$ we have

$$
\begin{aligned}
& \frac{1}{m(t)} D_{t}(s) \\
& =\frac{q}{q^{2}-1}\left(P_{\varepsilon}(t)-\frac{q-1}{q}\right)\left(P_{\varepsilon}(s)-\frac{q-1}{q}\right)+\frac{q^{2}}{q^{2}-1} P_{\varphi}(t) P_{\varphi}(s) \\
& \quad+\frac{q}{q-1} \sum_{\chi \in X^{\prime}\left(\mathbb{F}^{\times}\right) / \sim} P_{\chi}(t) P_{\chi}(s)+\frac{q}{q+1} \sum_{\nu \in X_{0}^{\prime}\left(\mathbb{K}^{\times}\right) / \sim} R_{\nu}(t) R_{\nu}(s) .
\end{aligned}
$$

Proof. This follows at once from Theorem 4.2 and elementary linear algebra.

Corollary 4.4. For all $v \in \mathbb{F}^{\times}, t \in \mathbb{F} \backslash\{ \pm 1\}$ and $\chi \in X^{\prime}\left(\mathbb{F}^{\times}\right)$we have

$$
\begin{gathered}
\mathbf{P}_{\pi(\varphi)}\left(\xi^{v \frac{1-t}{2}} \otimes \xi^{v \frac{1+t}{2}}\right)=-\frac{q}{q+1} P_{\varphi}(t) \mathbf{P}_{\pi(\varphi)}\left(\xi^{0} \otimes \xi^{v}\right) \\
\mathbf{P}_{\pi\left(\chi, \chi^{-1}\right)}\left(\xi^{v \frac{1-t}{2}} \otimes \xi^{v \frac{1+t}{2}}\right)=-\frac{q}{q+1} P_{\chi}(t) \mathbf{P}_{\pi\left(\chi, \chi^{-1}\right)}\left(\xi^{0} \otimes \xi^{v}\right) .
\end{gathered}
$$

Proof. If $\pi$ is any higher-dimensional irreducible representation of $G$ occurring in $\Pi$ then

$$
\mathbf{P}_{\pi}\left(\xi^{v \frac{1-t}{2}} \otimes \xi^{v \frac{1+t}{2}}\right)
$$

and $\mathbf{P}_{\pi}\left(\xi^{0} \otimes \xi^{v}\right)$ are both $\psi_{v}$-eigenvectors for the subgroup $N$ of $G$ and they also both lie in $V_{\pi}$. From the observations made in Section 3 we know that the space of such vectors is one-dimensional and from Theorem 4.1 it is clear that the second of the vectors is nonzero. Thus, for each $v, t$ and $\pi$, there is a constant $c(t, v, \pi)$ such that

$$
\mathbf{P}_{\pi}\left(\xi^{v^{\frac{1-t}{2}}} \otimes \xi^{v \frac{1+t}{2}}\right)=c(t, v, \pi) \mathbf{P}_{\pi}\left(\xi^{0} \otimes \xi^{v}\right) .
$$

The vector $\xi^{v \frac{1-t}{2}} \otimes \xi^{v \frac{1+t}{2}}$ is the sum of all its projections onto the various $V_{\pi}$ and writing out this relation and using (15) and Theorem 4.1 gives an expression for the function $D_{t}$ as a linear combination of the elements of $\mathcal{L}$, with coefficients involving the $c(t, v, \pi)$. But the unique such linear combination is given in Corollary 4.3 and by comparing the two one evaluates $c(t, v, \pi)$.

Since the Soto-Andrade sum $R_{\varphi \circ N}$ does not appear in the basis $\mathcal{L}$, it must be expressible as a linear combination of other Legendre and Soto-Andrade sums. The following result gives this expression.

Theorem 4.5. For all $a \in \mathbb{F}$ we have $R_{\varphi \circ N}(a)=-P_{\varphi}(a)$.

Proof. For $a= \pm 1$, this follows from Lemma 2.2 and the subsequent remarks. Thus we may assume that $a \neq \pm 1$. In the proof of Theorem 4.1 we calculated $\mathbf{P}_{\pi(\varphi)}\left(\xi^{0} \otimes \xi^{v}\right)$ by first calculating $\mathbf{P}_{\pi(\varphi, \varphi)}\left(\xi^{0} \otimes \xi^{v}\right)$ and then using (13). However, it is also possible to calculate this projection directly using (11) 
and the values of $\operatorname{ch}_{\pi(\varphi)}$. Suppose that $s \in \mathbb{F} \backslash\{0,1\}$ and $v \in \mathbb{F}^{\times}$. Then, calculating directly, we find that the coefficient of $\xi^{v(1-s)} \otimes \xi^{v s}$ in $\mathbf{P}_{\pi(\varphi)}\left(\xi^{0} \otimes\right.$ $\left.\xi^{v}\right)$ is

$$
\begin{aligned}
& \frac{1}{(q-1)^{2}(q+1)}\left[\sum_{(\alpha, \delta) \in \Omega^{+} / \sim} \varphi(\alpha \delta)\left\{-\frac{q+1}{q}-(q+1) \varphi\left(\alpha^{2}+\delta^{2}-2(2 s-1) \alpha \delta\right)\right\}\right. \\
& \left.-\sum_{z \in \Omega^{-} / \sim} \varphi \circ N(z)\left\{-\frac{q+1}{q}-(q+1) \varphi\left(S(z)^{2}-4 s N(z)\right)\right\}\right] .
\end{aligned}
$$

Removing the equivalence relations and completing both of the resulting sums we find that the coefficient is

$$
\begin{aligned}
& -\frac{1}{2(q-1)^{2}}\left[\sum_{\alpha, \delta \in \mathbb{F}^{\times}} \varphi(\alpha \delta) \varphi\left(\alpha^{2}+\delta^{2}-2(2 s-1) \alpha \delta\right)\right. \\
& \left.\quad-\sum_{z \in \mathbb{K}^{\times}} \varphi \circ N(z) \varphi\left(S(z)^{2}-4 s N(z)\right)\right],
\end{aligned}
$$

which evaluates to

$$
-\frac{q}{2(q-1)}\left[P_{\varphi}(2 s-1)-R_{\varphi \circ N}(2 s-1)\right] .
$$

The value of this coefficient found in Theorem 4.1 was $-\frac{q}{q-1} P_{\varphi}(2 s-1)$. Equating the two expressions we obtain $P_{\varphi}(2 s-1)=-R_{\varphi \circ N}(2 s-1)$ for all $s \in \mathbb{F} \backslash\{0,1\}$. This completes the proof.

\section{A characterization of the span of the Soto-Andrade sums.}

In this section we characterize the span of the set

$$
\mathcal{L}_{-}=\left\{R_{\nu} \mid \nu \in X_{0}^{\prime}\left(\mathbb{K}^{\times}\right) / \sim\right\}
$$

as the nullspace of a summation operator defined explicitly using Kloosterman sums. As a corollary of this result we evaluate the rank of the matrix of products of Kloosterman sums mentioned in the introduction.

Let $\mathcal{R}$ be the subspace of $W \otimes W$ spanned by the isotypic subspaces of all the cuspidal representations occurring in $\pi(\varepsilon) \otimes \pi(\varepsilon)$. Let $(W \otimes W)_{0}$ be the subspace of $W \otimes W$ consisting of all $N$-fixed vectors and $\mathbf{P}_{0}: W \otimes W \rightarrow$ $(W \otimes W)_{0}$ be the corresponding orthogonal projection. Then

$$
\mathbf{P}_{0}(\zeta)=\frac{1}{q} \sum_{x \in \mathbb{F}} \Pi(\mathbf{n}(x)) \zeta
$$

and, consequently, $\mathbf{P}_{0}$ commutes with all the operators $\mathbf{P}_{\pi}$ introduced before. The following characterization of $\mathcal{R}$ is well-known.

Lemma 5.1. We have

$$
\mathcal{R}=\left\{\zeta \in W \otimes W \mid \mathbf{P}_{0}(\Pi(g) \zeta)=0 \forall g \in G\right\} .
$$


Proof. Since cuspidal representations do not have nonzero $N$-fixed vectors, one inclusion is clear. If $\zeta \notin \mathcal{R}$ then there is some irreducible, non-cuspidal representation $\pi$ such that $\mathbf{P}_{\pi}(\zeta) \neq 0$. We may choose a nonzero $N$-fixed vector $\zeta_{0} \in V_{\pi}$. The set $\left\{\mathbf{P}_{\pi}(\Pi(g) \zeta) \mid g \in G\right\}$ spans $V_{\pi}$ and so $\zeta_{0}$ is a linear combination of vectors from this set. Writing $\zeta_{0}$ in this way and then applying $\mathbf{P}_{0}$ to both sides of the expression we find that $\mathbf{P}_{0}(\Pi(g) \zeta) \neq 0$ for some $g \in G$.

If $f \in \ell^{2}(\mathbb{F}, m)$ then write

$$
\zeta_{f}=\sum_{s \in \mathbb{F}} f(s) \xi^{\frac{1-s}{2}} \otimes \xi^{\frac{1+s}{2}} .
$$

Lemma 5.2. The function $f \in \ell^{2}(\mathbb{F}, m)$ lies in the span of the set $\mathcal{L}_{-}$if and only if $\mathbf{P}_{0}\left(\Pi(\mathbf{w t}(v, 1)) \zeta_{f}\right)=0$ for all $v \in \mathbb{F}^{\times}$.

Proof. From Theorem 4.1 and the fact that $\zeta_{f}$ is a $\psi_{1}$-eigenvector it follows that $f$ is in the span of $\left\{R_{\nu}\right\}$ if and only if $\zeta_{f} \in \mathcal{R}$. According to Lemma 5.1, this happens if and only if $\mathbf{P}_{0}\left(\Pi(g) \zeta_{f}\right)=0$ for all $g \in G$. If $B$ denotes the subgroup of $G$ consisting of upper-triangular matrices then $G=B \cup N \mathbf{w} B$ (the Bruhat decomposition). Certainly $\mathbf{P}_{0}\left(\Pi(b) \zeta_{f}\right)=0$ for all $b \in B$ and so the condition reduces to $\mathbf{P}_{0}\left(\pi(g) \zeta_{f}\right)=0$ for all $g \in N \mathbf{w} B$. Since $\mathbf{P}_{0}$ commutes with the action of $N$, the center of $G$ acts trivially via $\Pi$ and $\mathbf{n}(x) \zeta_{f}=\psi(x) \zeta_{f}$ for all $x \in \mathbb{F}$, this further reduces to the stated condition.

For $v \in \mathbb{F}^{\times}$let $\lambda_{v} \in \ell^{2}(\mathbb{F}, m)$ be the function defined by

$$
\lambda_{v}(s)=K\left(\frac{1}{2} v(1-s)\right) K\left(-\frac{1}{2} v(1+s)\right) .
$$

Functions of this form were encountered in the classical setting by Bateman in his study [1] of a family of polynomials generalizing the Legendre polynomials (see particularly pp. 118-119). Using $\lambda_{v}$ as a kernel, we define a summation operator $\Lambda$ on $\ell^{2}(\mathbb{F}, m)$ by

$$
\Lambda[f](v)= \begin{cases}\sum_{s \in \mathbb{F}} f(s) & \text { if } v=0, \\ \sum_{s \in \mathbb{F}} \lambda_{v}(s) f(s) m(s) & \text { if } v \neq 0 .\end{cases}
$$

We will use the operator $\Lambda$ to characterize the subspace of $\ell^{2}(\mathbb{F}, m)$ spanned by the set $\mathcal{L}_{-}$.

Lemma 5.3. Suppose that $f \in \ell^{2}(\mathbb{F}, m)$ and that $\Lambda[f](v)=0$ for all $v \in \mathbb{F}^{\times}$. Then $\Lambda[f](0)=0$ also.

Proof. Inserting the definition of the Kloosterman sums and interchanging the order of summation, one verifies that

$$
\sum_{v \in \mathbb{F}^{\times}} K\left(\frac{1}{2} v(1-s)\right) K\left(-\frac{1}{2} v(1+s)\right)= \begin{cases}-\frac{1}{q^{2}} & \text { if } s= \pm 1 \\ -\frac{(q+1)}{q^{2}} & \text { if } s \neq \pm 1 .\end{cases}
$$


Using Lemma 2.1, this may be expressed as

$$
\sum_{v \in \mathbb{F}^{\times}} \lambda_{v}(s)=\frac{1}{q}\left(P_{\varepsilon}(s)-\frac{q-1}{q}\right) .
$$

But $\left(P_{\varepsilon}(s)-\frac{q-1}{q}\right) m(s)=-(q+1) / q$ for all $s \in \mathbb{F}$ and so

$$
\sum_{v \in \mathbb{F}^{\times}} \Lambda[f](v)=-\frac{(q+1)}{q^{2}} \Lambda[f](0) .
$$

Theorem 5.4. The function $f \in \ell^{2}(\mathbb{F}, m)$ lies in the span of the set $\mathcal{L}_{-}$if and only if $\Lambda[f](v)=0$ for all $v \in \mathbb{F}^{\times}$.

Proof. Using (9) and the definition of $\zeta_{f}$ a routine calculation shows that

$$
\mathbf{P}_{0}\left(\Pi(\mathbf{w t}(v, 1)) \zeta_{f}\right)=\frac{1}{q^{2}} \sum_{s \in \mathbb{F}} f(s) \xi^{0} \otimes \xi^{0}+\sum_{b \in \mathbb{F}^{\times}, s \in \mathbb{F}} \lambda_{v b}(s) f(s) m(s) \xi^{b} \otimes \xi^{-b} .
$$

The claim follows on combining this with Lemmas 5.2 and 5.3.

Corollary 5.5. The sets $\left\{\lambda_{v} \mid v \in \mathbb{F}^{\times}\right\}$and

$$
\mathcal{L}_{+}=\left\{P_{\varepsilon}-\frac{q-1}{q}, P_{\varphi}, P_{\chi} \mid \chi \in X^{\prime}\left(\mathbb{F}^{\times}\right) / \sim\right\}
$$

span the same subspace of $\ell^{2}(\mathbb{F}, m)$.

Proof. Let $X$ be the subspace of $\ell^{2}(\mathbb{F}, m)$ spanned by $\left\{\lambda_{v} \mid v \in \mathbb{F}^{\times}\right\}$. Suppose that $f \in \ell^{2}(\mathbb{F}, m)$. Then, by definition, $\Lambda[\bar{f}](v)=\left\langle\lambda_{v}, f\right\rangle$ and so, applying Theorem 5.4, $f \in X^{\perp}$ if and only if $\bar{f}$ lies in the span of the set $\mathcal{L}_{\text {-. }}$. But the Soto-Andrade sums are real-valued and so the space they span is stable under complex conjugation. Thus $X^{\perp}$ is equal to the span of the set $\mathcal{L}_{-}$. Theorem 4.2 implies that $\operatorname{span}\left(\mathcal{L}_{-}\right)$is the orthogonal complement of $\operatorname{span}\left(\mathcal{L}_{+}\right)$and the assertion follows.

Corollary 5.6. Order the elements of $\mathbb{F}$, choose $a \in \mathbb{F}^{\times}$, and let

$$
M=[K(v t) K(v(t-a))]_{v \in \mathbb{F}^{\times}, t \in \mathbb{F}}
$$

be the indicated $(q-1)$-by-q matrix. Then the rank of $M$ is $(q+1) / 2$.

Proof. It follows from Corollary 5.5 that the rowspace of the matrix

$$
[K(v(1-s)) K(-v(1+s))]_{v \in \mathbb{F}^{\times}, s \in \mathbb{F}}
$$

is the subspace of $\ell^{2}(\mathbb{F}, m)$ spanned by $\mathcal{L}_{+}$. In fact, $\mathcal{L}_{+}$is a basis for this subspace and so its dimension is $(q+1) / 2$. Replacing $v$ by $a v / 2$ and $s$ by $1-2 t / a$ permutes the rows and columns of the matrix (16) and carries it to the matrix $M$. Thus the rank of $M$ is $(q+1) / 2$. 


\section{A summation identity.}

The representation-theoretic framework for the Legendre sums developed above can be used to derive a number of interesting identities involving Legendre sums, Soto-Andrade sums and generalized Kloosterman sums. In this section we prove one such identity. Our method will be to compute certain of the projections evaluated indirectly in Corollary 4.4 directly and then equate the results.

Theorem 6.1. For all $t \in \mathbb{F} \backslash\{ \pm 1\}, s \in \mathbb{F}$ and $\chi \in X\left(\mathbb{F}^{\times}\right) \backslash\{\varepsilon\}$ we have

$$
\begin{aligned}
\sum_{x \in \mathbb{F}^{\times}} \chi(x)^{-1} K\left(\frac{(1-t)(1-s)}{4} x\right) K\left(\frac{(1+t)(1+s)}{4} x\right) K_{\chi^{2}}(x) & \\
& =P_{\chi}(s) P_{\chi}(t)-\frac{1}{q} D_{t}(s) .
\end{aligned}
$$

Proof. Fix $t \in \mathbb{F} \backslash\{ \pm 1\}$ and $s \in \mathbb{F}$. Our first step is to find the coefficient of $\xi^{\frac{1-s}{2}} \otimes \xi^{\frac{1+s}{2}}$ in $\Pi(c)\left(\xi^{\frac{1-t}{2}} \otimes \xi^{\frac{1+t}{2}}\right)$ for each conjugacy class $c \in \mathcal{C}$ such that $\operatorname{ch}_{\pi\left(\chi, \chi^{-1}\right)}(c) \neq 0$. The calculations are similar to those in the proof of Theorem 4.1 and the results are as follows. The coefficient of $\xi^{\frac{1-s}{2}} \otimes \xi^{\frac{1+s}{2}}$ in $\Pi(c)\left(\xi^{\frac{1-t}{2}} \otimes \xi^{\frac{1+t}{2}}\right)$ is $D_{t}(s)$ if $c=C(\mathbf{t}(\alpha, \alpha))$,

$$
-D_{t}(s)+q \sum_{b \in \mathbb{F}^{\times}} K\left(\frac{(1-t)(1-s)}{4} \alpha^{2} b^{2}\right) K\left(\frac{(1+s)(1+t)}{4} \alpha^{2} b^{2}\right) \psi(2 \alpha b),
$$

if $c=C\left(\mathbf{t}(\alpha, \alpha) \mathbf{n}\left(\alpha^{-1}\right)\right)$ and

$$
q \sum_{x \in \mathbb{F}^{\times}} K\left(\frac{(1-t)(1-s)}{4} \alpha \delta x^{2}\right) K\left(\frac{(1+t)(1+s)}{4} \alpha \delta x^{2}\right) \psi((\alpha+\delta) x)
$$

if $c=C(\mathbf{t}(\alpha, \delta))$.

Using these values, we proceed to calculate the coefficient of $\xi^{\frac{1-s}{2}} \otimes \xi^{\frac{1+s}{2}}$ in $\mathbf{P}_{\pi\left(\chi, \chi^{-1}\right)}\left(\xi^{\frac{1-t}{2}} \otimes \xi^{\frac{1+t}{2}}\right)$ and find it to be

$$
\begin{array}{r}
\frac{1}{q-1} D_{t}(s)+\frac{1}{(q-1)^{2}} \sum_{\alpha, \delta, x \in \mathbb{F}^{\times}} \chi\left(\frac{\alpha}{\delta}\right) K\left(\frac{(1-t)(1-s)}{4} \alpha \delta x^{2}\right) \\
K\left(\frac{(1+t)(1+s)}{4} \alpha \delta x^{2}\right) \psi((\alpha+\delta) x) .
\end{array}
$$


In this sum we eliminate the variable $\alpha$ in favor of $y=\alpha / \delta$, replace $x$ by $\delta^{-1} x$ and $y$ by $\delta^{-2} y$ to obtain

$$
\begin{aligned}
& \frac{1}{q-1} D_{t}(s)+\frac{q}{q-1} \sum_{y \in \mathbb{F}^{\times}} \chi(y) K\left(\frac{(1-s)(1-t)}{4} y\right) \\
& \cdot K\left(\frac{(1+s)(1+t)}{4} y\right) K_{\chi^{-2}}(y) .
\end{aligned}
$$

Since $\pi\left(\chi, \chi^{-1}\right) \cong \pi\left(\chi^{-1}, \chi\right)$, we may replace $\chi$ by $\chi^{-1}$ in this expression without altering its value. Suppose that $\chi \in X^{\prime}\left(\mathbb{F}^{\times}\right)$. Then, combining (14) and Corollary 4.4, we see that this coefficient is also equal to

$$
-\frac{q}{q+1} P_{\chi}(t) \cdot-\frac{q+1}{q-1} P_{\chi}(s)=\frac{q}{q-1} P_{\chi}(s) P_{\chi}(t)
$$

and equating this with the above expression gives the required identity. If $\chi=\varphi$ then using (13), (14) and Corollary 4.4 gives the same conclusion.

One can easily extend Theorem 6.1 to include the case where $\chi=\varepsilon$, but this is uninteresting, since it isn't hard to evaluate

$$
\sum_{x \in \mathbb{F}^{\times}} K(A x) K(B x) K(C x)
$$

for general $A, B, C \in \mathbb{F}^{\times}$(see Equation (3.20) in [7]).

Corollary 6.2. Suppose that $q \equiv 1$ (4) and that $\lambda \in X\left(\mathbb{F}^{\times}\right)$satisfies $\lambda^{2}=$ $\varphi$. Then, for all $t \in \mathbb{F} \backslash\{ \pm 1\}$ and $s \in \mathbb{F}$,

$$
\begin{aligned}
& \sum_{x \in \mathbb{F}^{\times}} \varphi(2 x) K\left((1-t)(1-s) x^{2}\right) K\left((1+t)(1+s) x^{2}\right) \psi(4 x) \\
& =\Gamma(\varphi)\left(P_{\lambda}(s) P_{\lambda}(t)-\frac{1}{q} D_{s}(t)\right) .
\end{aligned}
$$

Proof. This follows from Theorem 6.1 on substituting $\chi=\lambda$ and then using the well-known evaluation of the Salié sum $K_{\varphi}$.

\section{References}

[1] H. Bateman, A generalization of the Legendre polynomial, Proc. London Math. Soc. (2), 3 (1905), 111-123.

[2] R.J. Evans, Hermite character sums, Pacific J. Math., 122 (1986), 357-390, MR 87e:11140, Zbl 0603.12010.

[3] _ Character sums as orthogonal eigenfunctions of adjacency operators for Cayley graphs, in 'Finite Fields: Theory, Applications, and Algorithms', Contemporary Mathematics, 168, 33-50, Amer. Math. Soc., Providence, 1994, MR 95g:11121, Zbl 0817.11058. 
[4] I.M. Gelfand, M.I. Graev and I.I. Piatetski-Shapiro, Representation Theory and Automorphic Functions, Academic Press, San Diego, 1990, MR 91g:11052, Zbl 0718.11022.

[5] J. Greene, Hypergeometric functions over finite fields, Trans. Amer. Math. Soc., 301 (1987), 77-101, MR 88e:11122, Zbl 0629.12017.

[6] _ Hypergeometric functions over finite fields and representations of $S L(2, q)$, Rocky Mountain J. Math., 23 (1993), 547-568, MR 94g:33012, Zbl 0806.11062.

[7] D.H. Lehmer and Emma Lehmer, The cyclotomy of Kloosterman sums, Acta Arith., 12 (1967), 385-407, MR 35 \#5413, Zbl 0149.28603.

[8] K. Ono, Values of Gaussian hypergeometric series, Trans. Amer. Math. Soc., 350 (1998), 1205-1223, MR 98e:11141, Zbl 0910.11054.

[9] I. Piatetski-Shapiro, Complex Representations of $G L(2, K)$ for Finite Fields $K$, Contemporary Mathematics, 16, Amer. Math. Soc., Providence, 1983, MR 84m:20046, Zbl 0513.20026.

[10] Y. Sawabe, Legendre character sums, Hiroshima Math. J., 22 (1992), 15-22, MR 93i:11147, Zbl 0752.11056.

[11] J. Soto-Andrade, Geometric Gel'fand models, tensor quotients, and Weil representations, in 'The Arcata Conference on Representations of Finite Groups', Proc. Symp. Pure Math., 47, 305-316, Amer. Math. Soc., Providence, 1987, MR 89h:20058, Zbl 0652.20047.

[12] N.Ja. Vilenkin and A.U. Klimyk, Representations of Lie Groups and Special Functions, Volume 1: Simplest Lie Groups, Special Functions and Integral Transforms, Mathematics and Its Applications (Soviet Series), 72, Kluwer Academic Publishers, Dordrecht, 1991, MR 93h:33009, Zbl 0742.22001.

Received November 28, 2000 and revised November 21, 2001.

Department of Mathematics

OKlahoma State University

STILLWATER OK 74078

E-mail address: akable@math.okstate.edu 